\title{
Primera Radiocirugía de schwannoma vestibular en el sistema público uruguayo, Hospital Regional de Tacuarembó. Centro Oncológico del Norte.
}

First Radiosurgery for vestibular schwannoma in the Uruguayan public system, Hospital Regional de Tacuarembó. North Cancer Center.

Primeira radiocirurgia para schwannoma vestibular da rede pública uruguaia, Hospital Regional de Tacuarembó. North Cancer Center.

Leandro Ricagni Resumen: Los schwannomas vestibulares son tumores benignos de lento crecimiento. Aproximadamente el $90 \%$ son de presentación unilateral, el $10 \%$ restante, son bilaterales y se encuentran asociados al síndrome de Neurofibromatosis tipo 2. Las opciones de tratamiento son observación, cirugía o radiocirugía. La radiocirugía es un tratamiento seguro, con altas tasas de control local y baja morbilidad en schwannomas vestibulares menores a $3 \mathrm{~cm}$ de diámetro mayor. Reportamos la primera radiocirugía realizada en el sistema público uruguayo para este tipo de patologías.

Milexis Rivero ORCID: 0000-0002-1697-0904 Postgrado Oncología Radioterápica. Hospital de Clínicas.

Pablo Pereda ORCID: 0000-0001-6273-4059

Neurocirujano. Jefe Servicio Neuroquirúrgico Hospital Regional de Tacuarembó.

Aldo Quarneti ORCID: 0000-0002-7861-6142 Profesor Titular Cátedra Oncología Radioterápica. Hospital de Clínicas.

Palabras clave: Schwannoma vestibular, radiocirugìa

Abstract: Vestibular schwannomas are slow-growing benign tumors. Approximately $90 \%$ are unilateral, the remaining $10 \%$ are bilateral and are associated with Neurofibromatosis type 2 syndrome. Treatment options are observation, surgery or radiosurgery. Radiosurgery is a safe treatment, with high local control rates and low morbidity in vestibular schwannomas smaller than $3 \mathrm{~cm}$ in major diameter. We report the first radiosurgery performed in the Uruguayan public system for this type of pathology.

Key words: Vestibular Schwannoma, radiosurgery.

Resumo: Os schwannomas vestibulares são tumores benignos de crescimento lento. Aproximadamente $90 \%$ são unilaterais, os restantes $10 \%$ são bilaterais e estão associados à síndrome de neurofibromatose tipo 2 . As opções de tratamento são observação, cirurgia ou radiocirurgia. A radiocirurgia é um tratamento seguro, com altas taxas de controle local e baixa morbilidade em schwannomas vestibulares com menos de $3 \mathrm{~cm}$ de diâmetro maior. Relatamos a primeira radiocirurgia realizada no sistema público uruguaio pra este tipo de patologia.

Palavras-chave: Schwannoma vestibular, radiocirurgia.

Recibido: 15/06/2021 - Aceptado: 23/09/2021

Instituto Centro Oncológico del Norte. Hospital Regional de Tacuarembó. Uruguay.

Correspondencia. E-mail: leandroricagni@gmail.com 


\section{Introducción}

Los schwannomas vestibulares (SV), comúnmente denominados neurinomas del acústico, surgen de las células de Schwann de la rama vestibular del octavo nervio craneal (nervio acústico), son tumores cerebrales benignos de crecimiento lento que afectan la calidad de vida del paciente ${ }^{(1)}$.

La incidencia de SV ha ido en aumento en los últimos años y se aproxima a 20 casos por millón habitantes por año, debido a factores como, una mayor conciencia entre los médicos sobre el diagnóstico de SV, un mayor acceso a tomografía computada y resonancia nuclear magnética, y mayores pruebas de diagnóstico en pacientes sintomáticos. Representan el 80$90 \%$ de los tumores del ángulo pontocerebeloso. El pico de incidencia es en la quinta década de la vida, y presentan igual proporción en hombres y mujeres ${ }^{(2)}$.

El SV unilateral es la forma más común de presentación, hasta un 95\% de los casos. Se asocian en pacientes jóvenes a la mutación del gen neurofibromatosis 2 (NF2), presentando un alto riesgo de desarrollar afección bilateral. Además, estos pacientes tienen un mayor riesgo de desarrollar otros tumores del sistema nervioso y de transformación maligna ${ }^{(3)}$.

En los SV de reciente diagnóstico, dependiendo de su tamaño, sintomatología, relación con tronco encefálico, terreno y edad del paciente, se pueden plantear varias posibles conductas que van desde el seguimiento imagenológico (en SV chicos, asintomáticos o pacientes con un muy mal terreno) hasta la cirugía, siendo la radiocirugía estereotáctica (RC) una opción de tratamiento en casos seleccionados.

La $\mathrm{RC}$ consiste en la administración precisa de una dosis de radiación terapéuticamente efectiva, en una sola sesión, a un objetivo definido por imágenes ${ }^{(4)}$.

Las indicaciones de RC incluyen SV recientemente diagnosticados en pacientes no candidatos a resección quirúrgica o que no optan por la cirugía, SV residuales después de cirugía y SV recurrentes. Los pacientes deben presentarse sin compresión sintomática del tronco encefálico (Koos igual o menor a 3), y el tamaño del tumor debe ser inferior a $3 \mathrm{~cm}$ de diámetro ${ }^{(5)}$.

La RC es una técnica segura y con alto control tumoral, Kondziolka et al. en un seguimiento a largo plazo de 162 pacientes tratados con SRS con una dosis marginal tumoral de 16 Gy, reporta una tasa de control tumoral del $98 \%$, con una función facial conservada en el $79 \%$ (House-Brackmann G1), y sin cambios en capacidad auditiva en un $51 \%$. ${ }^{(6)}$ Dado el alto control obtenido con esta técnica, en los años subsiguientes se estudiaron nuevas dosis para obtener un mejor balance entre control tumoral y efectos secundarios. Chopra et al. reportan en una serie de 216 pacientes tratados con SRS con una dosis marginal tumoral de 12 a 13 Gy el mismo control tumoral (98.3\%), mejorando las cifras de preservación auditiva (78,6\%), y preservación funcional del VII par (100\%), estableciéndose hasta el día de hoy como las dosis estándar para $\mathrm{RC}$ fracción única en SV ${ }^{(7,8)}$.

El objetivo de esta publicación es presentar el primer caso de SV tratado con RC en el medio público en Uruguay, y hacer una breve revisión del tema.

\section{Caso Clínico}

Paciente de sexo femenino, de 67 años de edad, con antecedentes personales de cirugía por tumor del ángulo ponto cerebeloso izquierdo hace 18 años. Se desconoce anatomía patológica. Sordera izquierda secuelar. Presenta hipoacusia en oído derecho y vértigo paroxístico postural. Se solicita resonancia nuclear magnética (RNM) de cráneo que reporta proceso expansivo del ángulo ponto cerebeloso derecho de $15 \mathrm{~mm}$ de eje mayor, con una porción cisternal de $8 \mathrm{~mm}$ y el resto intracanalicular. No ejerce efecto de masa. Figura 1. Diagnóstico imagenológico de Schwannoma vestibular derecho grado 2 por la clasificación de Koos. Tabla 1.

\begin{tabular}{|c|c|}
\multicolumn{2}{|c|}{ Clasificación de Koos } \\
\hline Grado 1 & Tumor intracanalicular \\
\hline Grado 2 & $\begin{array}{c}\text { Tumor pequeño con protrusión hacia la cisterna cerebropontina, no contacta con } \\
\text { tronco encefálico }\end{array}$ \\
\hline Grado 3 & Tumor que ocupa cisterna cerebro pontina sin desplazar tronco encefálico \\
\hline Grado 4 & Tumor grande con desplazamiento de tronco encefálico y/o nervios craneales \\
\hline
\end{tabular}


Figura 1: RNM secuencia T1 con Gadolineo. Se observa Schwannoma

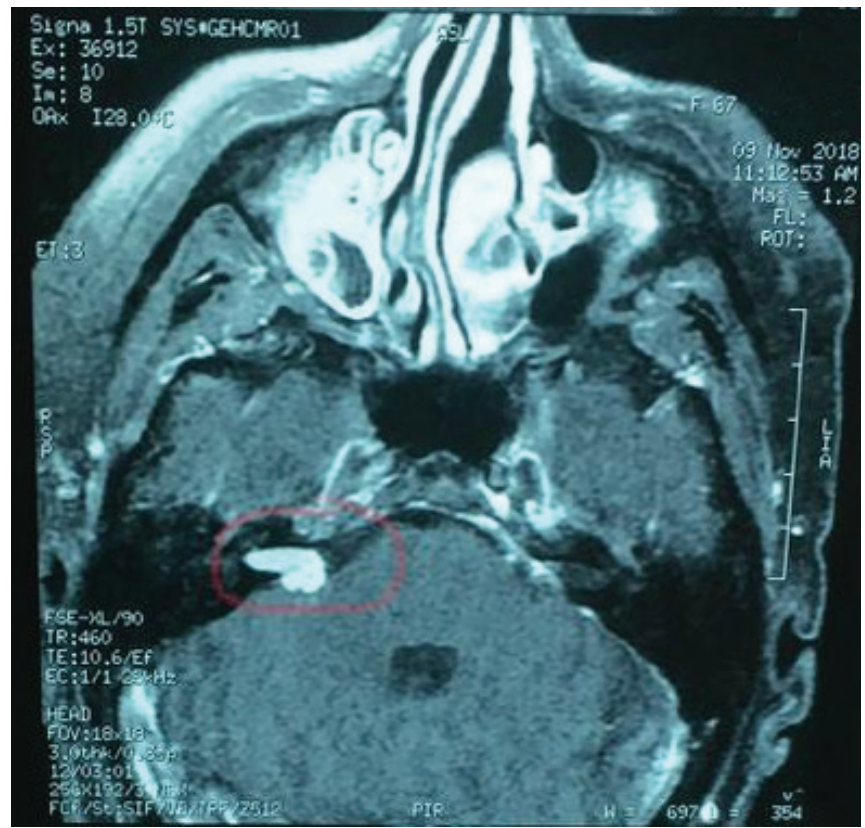

La paciente llega a nuestra consulta buscando una técnica que pueda controlar el tumor y que le permita mantener audición ya que presenta cofosis en su oído izquierdo. Dadas las características de la lesión se plantea RC fracción única.

\section{c) Técnica - RC fracción única}

La paciente fue inmovilizada con un marco estereotáctico con sistema de fiduciales radiolúcidos referenciales lo que permite localizar un objetivo en los tres planos del espacio. Se realizó tomografía computada de cortes finos con contraste para la identificación de la lesión. Figura 2

Figura 2: Realización de Tomografia de

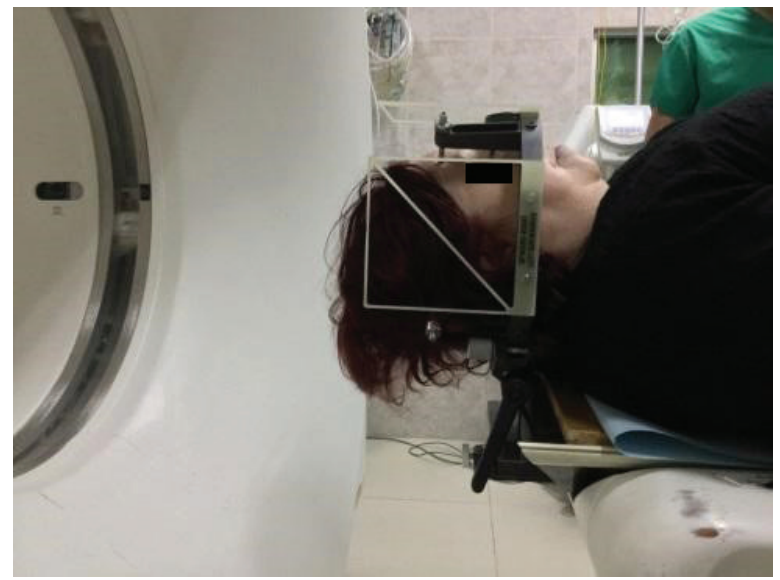

Las imágenes se ingresaron en formato DICOM a un sistema de planificación TPS (por sus siglas en inglés Treatment Planning System), MIRS versión 5.2, para delimitar los volúmenes objetivos y seleccionar la entrada y trayectoria de los haces de fotones de 6 MegaVoltios.

El volumen tumoral blanco GTV (de sus siglas en inglés Gross Tumor Volume) debe definirse como el realce tumoral del contraste en las imágenes de resonancia magnética ponderada en T1 o en tomografía computada. No agregamos margen al GTV para conformar el volumen blanco de planificación PTV (por sus siglas en inglés Planning Target Volumen), volumen que contempla un posible error geográfico, ya que el marco estereotáctico tiene una precisión submilimétrica. Este punto es debatible se puede agregar un margen de $1 \mathrm{~mm}$ como PTV. ${ }^{(9)}$

Para la delimitación del GTV puede realizarse fusión de la resonancia magnética de diagnóstico con la tomografía de planificación si el planificador cuenta con dicha herramienta. Se contornearon los órganos en riesgo OARs (por sus siglas en inglés organs at risk) que deben ser preservados de las curvas de dosis altas que puedan generar daño a su función. Para 
Figura 3: Posicionamiento y confirmacion de coordenadas en marco

de referencia Acelerador Lineal Clinac 600. Cabezal con Sistema de Conos para colimacion del haz.

Figura 4: Cortes axial, coronal y sagital. GTV, curva de isodosis de prescripción (12Gy), y OARs.

nuestro caso los OARs fueron: globos oculares, cristalinos, nervios ópticos, quiasma óptico, hipófisis, cóclea, tronco encefálico.

Se realizó una técnica con isocentro único con 6 arcos no coplanares con colimación del haz con Sistema de Conos. Se seleccionaron conos de $18 \mathrm{~mm}$. El procedimiento se realizó en un Acelerador Lineal Varian Clinac 600. Figura 3

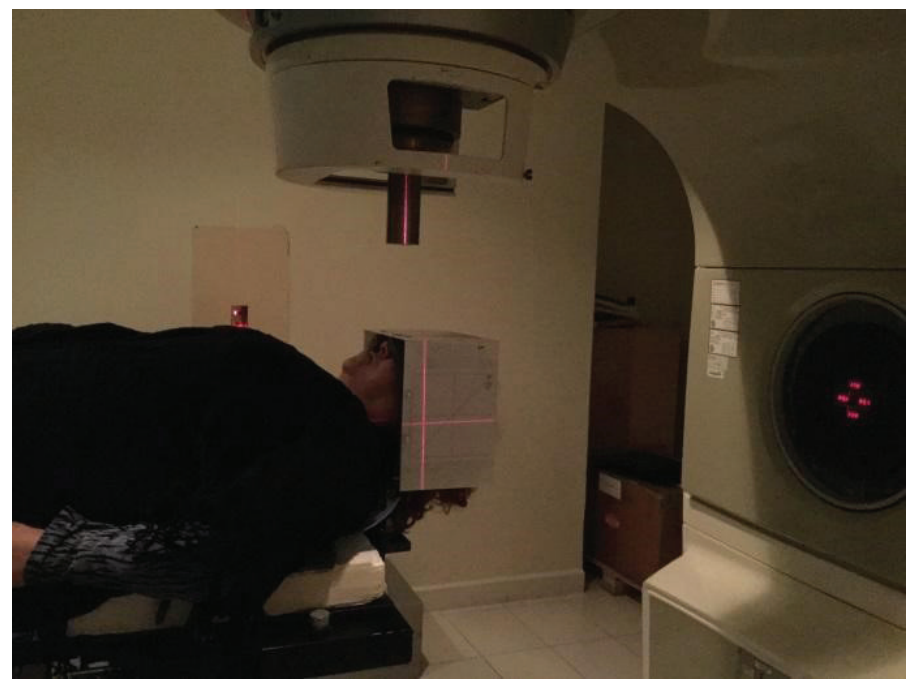

La lesión fue cubierta por la curva de isodosis de $80 \%$ normalizada en 12 Gy. Se obtuvieron puntos de máxima dosis de 15,03 Gy dentro del GTV y la lesión recibió un promedio de 14,28 Gy; la cóclea, principal OAR, recibió una dosis máxima de 7,09 GY. Figura 4.

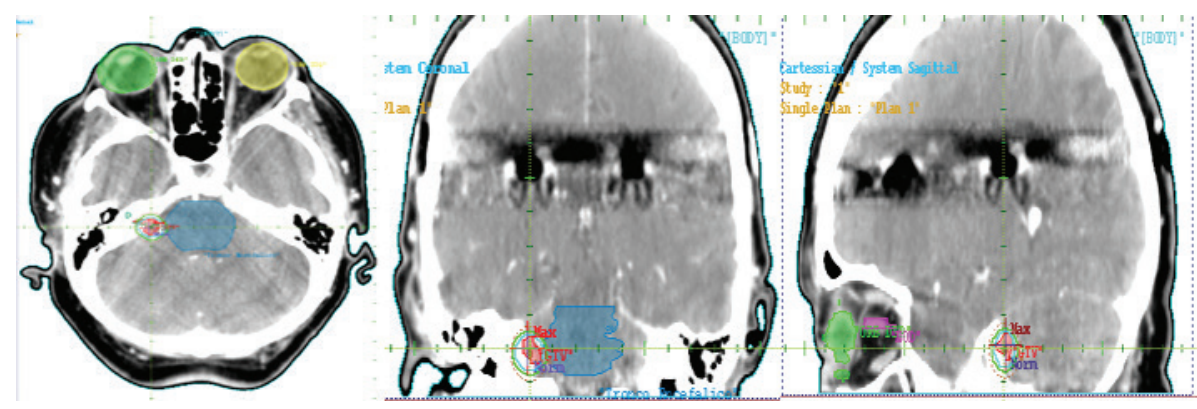

Estos parámetros son estudiados en el histograma dosis volumen (DVH por sus siglas en inglés Dose-Volume Histogram) Figura 5.

\begin{tabular}{|c|c|c|c|c|c|c|c|c|}
\hline \multicolumn{9}{|c|}{ DVH additional evaluation } \\
\hline \multirow{2}{*}{ Regions } & \multirow{2}{*}{ Volume [ce] } & \multicolumn{3}{|c|}{ Plan : Plan 1} & \multicolumn{4}{|l|}{$\checkmark$} \\
\hline & & Dmin[cGy] & |Dmax[cGy] & Davg(cGy) & DVI[cGy.ce] & EUD1[cGy] & EUD2[cGy] & Alarms \\
\hline [BODY] & 2643.19 & o.0 & 1503.4 & 27.5 & 72693.8 & \begin{tabular}{|c|} 
0.0 \\
\end{tabular} & 657.7 & Oen \\
\hline GTV & o.39 & 986.9 & 1503.4 & 1428.6 & 553.9 & 1387.2 & 1442.1 & 00 \\
\hline OAB DEA & 10.15 & 0.0 & 5.1 & 1.3 & 12.9 & 0.0 & 3.2 & 0 en \\
\hline ORB IZQ & 8.92 & 0.3 & 5.5 & 1.9 & 16.8 & $\mathbf{0 . 5}$ & $\mathbf{3 . 3}$ & 00 \\
\hline NOD & 0.71 & 0.2 & 9.3 & 3.5 & 2.5 & 0.4 & 6.2 & $0=$ \\
\hline NOI & 1.08 & 2.8 & 25.8 & 7.9 परे & 8.5 & 4.5 & 16.1 & 00 \\
\hline hipofisis & 0.83 & 30.6 & 121.2 & 64.7 & 53.5 & 45.5 & 84.0 & 00 \\
\hline Quiasma & 1.59 & 8.0 & 113.2 & 51.5 & 82.1 & 16.9 & 78.1 & 00 \\
\hline Coclea & 0.13 & 212.9 & 709.4 & 485.7 & 64.4 & 347.5 & 547.4 & 00 \\
\hline Tronco Encefalico & 12.55 & 50.0 & 1317.4 & 229.1 & 2876.2 & 90.9 & 711.7 & Cese \\
\hline
\end{tabular}

\section{d) Resultado}

El seguimiento de la paciente fue clínico e imagenológico. Se realizaron RNM de cráneo cada 6 meses luego de RC. Figura 6 . Nótese en la primera y segunda RM se observa la lesión con centro hipodenso en relación a los cambios pos RC. El tamaño tumoral se ha mantenido incambiado en los estudios realizados.

Realizamos audiograma de control a los 2 años de la radiocirugía el cual demuestra que la agudeza auditiva del oído derecho se mantiene exactamente igual (hipoacusia moderada), 
presentado la paciente un deterioro en los valores de la logo-audiometría (discriminación de la palabra).

Figura 6: Secuencia cronológica de controles con RNM pos RC. Se pueden observar los cortes axiales en la secuencia $\mathrm{T} 1$ con contraste. De izquierda a derecha y octubre 2020 y abril 2021.

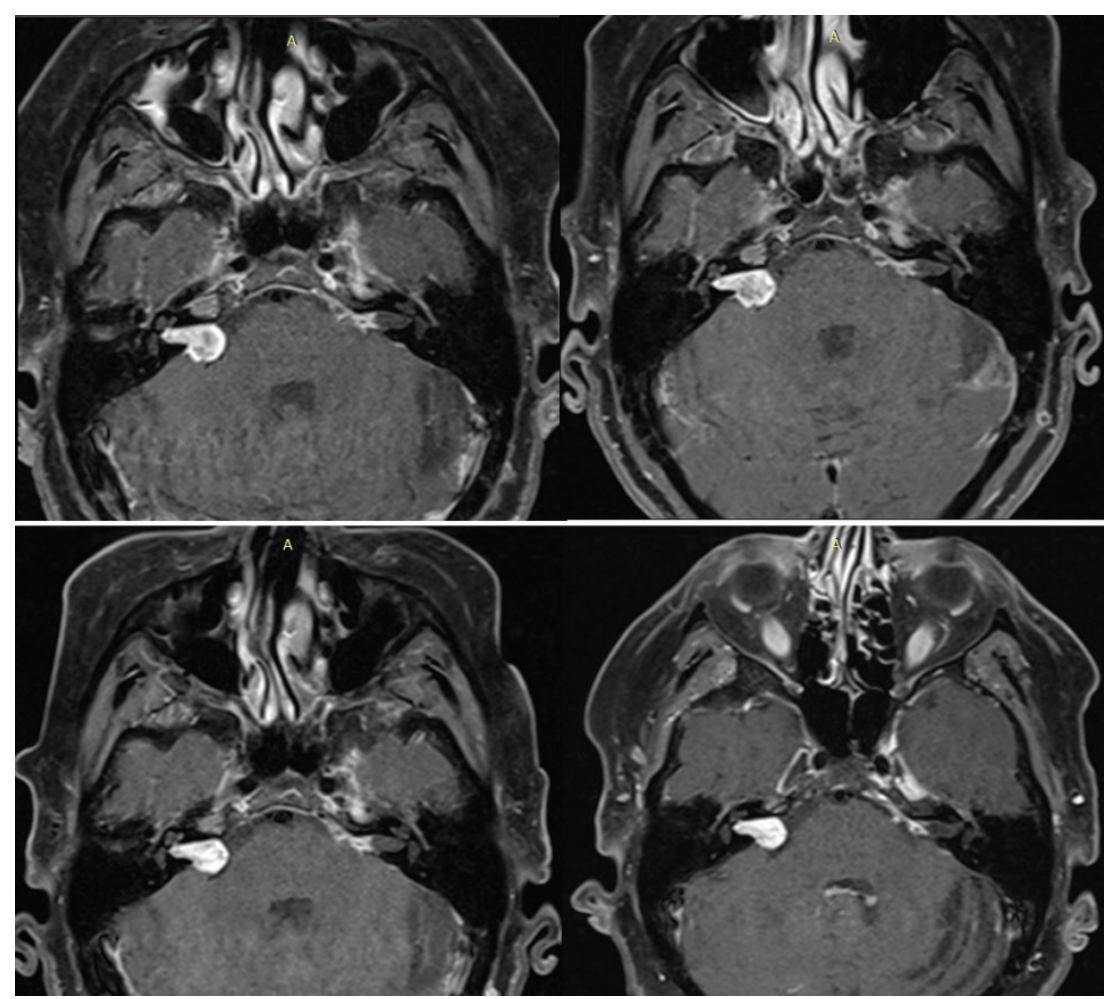

La paciente mantiene elementos de síndrome vestibular como inestabilidad en la marcha con tendencia a la latero-pulsión a derecha, de leve intensidad, que no afecta las tareas diarias. Mantiene una función completamente conservada del nervio facial derecho.

\section{Discusión}

Los SV son tumores benignos, de lento crecimiento, que se originan en las células de Schwann de la rama vestibular del octavo par craneano. Son los tumores más frecuentes de la región anatómica denominada ángulo ponto cerebeloso (APC), tienen característicamente una porción intracanalicular, una de las principales características que los diferencian de los meningiomas, segundos tumores en frecuencia del APC.

Las opciones terapéuticas para estos tumores son, la observación y seguimiento, la microcirugía, y/o la radiación.

La observacion y seguimiento puede ser planteada en tumores pequeños, grados 1 o 2 de la clasificacion de Koos y asintomáticos. En tumores sintomaticos o en los cuales se ha documentado un crecimiento sostenido, las terapias intervencionistas pueden ser planteadas. En este escenario, en tumores que no compriman o desplacen el tronco encefálico grados 1, 2, 3 de la clasificación de Koos, se puede realizar un abordaje microquirúrgico o radiante. En los tumores grandes que desplazan o comprimen tronco encefálico y/o pares craneanos, la microcirugía es mandatoria. En tumores gigantes donde la reseccion completa puede determinar grandes riesgos de secuelas auditivas o lesion del nervio facial, se puede realizar un tratamiento combinado de microcirugía para disminuir volumen tumoral y decomprimir estructuras, y luego completar el tratamiento al tumor remanente con radioterapia.

En nuestro caso, si bien no tenemos confirmación anatomopatológica del tumor del ángulo ponto cerebeloso izquierdo tratado hace años, considerando la edad de resección y la ubicación del tumor, sospechamos pueda haberse tratado de un SV que, sumado a éste, haría a la paciente portadora de SV bilaterales, situación que debe hacer sospechar la posibilidad de una neurofibromatosis tipo 2, aunque esto no es patognomónico, y no contamos con historia familiar de la enfermedad.

Como se analizó previamente las característias del tumor hacian plausible el abordaje con radiocirugia fraccion única o microcirugía. En este caso como la paciente presenta una acusia secuelar al tratamiento quirúrgico, optó por el tratamiento radiante. 
Los enfoques de radioterapia que se han utilizado en los SV incluyen radiocirugía estereotáctica fracción única $(\mathrm{RC})$, más recientemente la Radiocirugia esterotáctica fraccionada (RTF), la radioterapia externa de fraccionamiento convencional (RTE) y radioterapia con haz de protones (RTP). No hay ensayos prospectivos que comparen estos diferentes esquemas de tratamiento radiante, por lo tanto, la elección se basa en las características del paciente, la disponibilidad de la técnica y la experiencia del centro.

Para la RC, una dosis de 12-13 Gy presenta una tasa de control tumoral esperada > al $90 \%{ }^{(10)}$. Según reporte de estudios retrospectivos entre el $50-60 \%$ de los casos tratados con $\mathrm{RC}$ presentan disminución del tamaño tumoral, aproximadamente el $30 \%$ estabilidad y el resto pueden presentar aumento en el tamaño. En este último caso el aumento promedio son 3 $\mathrm{mm}$, asociado a una necrosis central, lo que no se considera fracaso terapéutico, pues este incremento es en la mayoría de casos transitorio con posterior estabilidad e incluso regresión en 1-2 años luego de la SRS. Por lo tanto solo después de este plazo y teniendo un crecimiento continuo o cambios sintomáticos ostensibles, podría plantearse una microcirugia. ${ }^{(11)}$

Los SV dentro del contexto de una NF2 son de mayor agresividad lo que puede afectar la efectividad de la técnica con respecto a los resultados en SV esporádicos. ${ }^{(12)}$ En nuestro caso la paciente presenta una estabilidad lesional a 2 años, según imágenes de RNM como se observó en la Figura 6.

Si bien la RC es un tratamiento seguro y de poca morbilidad, la función auditiva pretratamiento, el tamaño tumoral, la dosis a la cóclea y el contacto con el tronco encefálico, son variables asociadas a la declinación auditiva posterior a la RC. Aunque la toxicidad del nervio facial y trigémino con dosis de 12 Gy son bajas, $1.5 \%$ y $1.6 \%$, respectivamente, la hipoacusia es un punto importante. ${ }^{(13)}$ Alrededor de un $40 \%$ de los pacientes presentan pérdida de la audición dentro de los 2 años posteriores a la radioterapia..$^{(14-16)}$ Sin embargo, en pacientes bajo estrategia de "observación y espera" también se evidencia pérdida de audición, lo que hace pensar que este deterioro funcional observado después de la SRS pueda ser también resultado de los tumores que surgen del nervio vestibulococlear, y no directamente efectos adversos de la radiación. ${ }^{(16)}$. En cuanto a la dosis umbral de la cóclea se requieren datos más robustos, se sugiere calcular los volúmenes de 4, 6 y 8 Gy, (9) en la mayoría de los estudios la dosis sugerida está entre 4-5.33 Gy, pero Jacob et al. en el seguimiento de 105 pacientes no encontraron una asociación estadísticamente significativa con este parámetro ${ }^{(17)}$. La radiocirugía estereotáctica fraccionada presenta mayores tasas de preservación auditiva y está indicada también en SV de mayor tamaño. ${ }^{(18)}$

Lo que se considera prudente es no disminuir la dosis en el objetivo lo cual podría comprometer el control tumoral. La revisión hecha por Linskey, sugiere que la toxicidad en el VCN (núcleo coclear ventral), asociado a dosis $>9$ Gy, conduce preferentemente a una pérdida auditiva de frecuencias bajas, mientras que la toxicidad coclear a una pérdida principalmente de frecuencias auditivas altas. ${ }^{(19,20)}$

\section{Conclusiones}

La RC es una técnica eficaz y segura, en pacientes seleccionados. Obtiene tasas de control tumoral superiores al $90 \%$ con una dosis fracción de 12 Gy con toxicidades aceptables. Creemos de interés para la comunidad médica nacional, reportar el primer caso de SV tratado en el sistema público nacional, con 2 años de seguimiento, donde se obtuvo control lesional y buen resultado en cuanto a presevación de la función auditiva.

\section{Bibliografía}

1- Propp JM, McCarthy BJ, Davis FG, Preston-Martin S. Descriptive epidemiology of vestibular schwannomas. Neuro-oncology. 2006; 8(1): 1-11. https://doi.org/10.1215/S1522851704001097.

2- Stangerup SE, Caye-Thomasen $P$, Tos $M$, Thomsen J. The natural history of vestibular schwannoma. Otol Neurotol. 2006 Jun;27(4):547-52. doi: 10.1097/01.mao.0000217356.73463.e7.

3- Mohyuddin A, Neary WJ, Wallace A, Wu CL, Purcell S, Reid H, et al. Molecular genetic analysis of the NF2 gene in young patients with unilateral vestibular schwannomas. J Med Genet. 2002 May;39(5):315-22. doi: 10.1136/jmg.39.5.315.

4- Pollock BE, Lunsford LD. A call to define stereotactic radiosurgery. Neurosurgery. 2004 Dec;55(6):13713. doi: 10.1227/01.neu.0000143613.13759.d4

5- Golfinos JG, Hill TC, Rokosh R, Choudhry O, Shinseki M, Mansouri A, et al. A matched cohort comparison of clinical outcomes following microsurgical resection or stereotactic radiosurgery for 
Rev. urug. med. interna.

patients with small- and medium-sized vestibular schwannomas. J Neurosurg. 2016 Dec;125(6):14721482. doi: 10.3171/2015.12.JNS151857.

6- Kondziolka D, Lunsford LD, McLaughlin MR, Flickinger JC. Long-term outcomes after radiosurgery for acoustic neuromas. N Engl J Med. 1998 Nov 12;339(20):1426-33. doi: 10.1056/ NEJM199811123392003

7- Chopra R, Kondziolka D, Niranjan A, Lunsford LD, Flickinger JC. Long-term follow-up of acoustic schwannoma radiosurgery with marginal tumor doses of 12 to 13 Gy. Int J Radiat Oncol Biol Phys. 2007 Jul 1;68(3):845-51. doi: 10.1016/j.jijrobp.2007.01.001.

8- Massager N, Lonneville S, Delbrouck S, Benmebarek N, Desmedt F, Devriendt D. Dosimetric and Clinical Analysis of Spatial Distribution of the Radiation Dose in Gamma Knife Radiosurgery for Vestibular Schwannoma, International Journal of Radiation Oncology Biology Physics. 2011; 81(4): e511-e518. Disponible en: https://doi.org/10.1016/j.jijobp.2011.03.047

9- Mindermann T, Schlege I. Grading of Vestibular Schwannomas and Corresponding Tumor Volumes: Ramifications for Radiosurgery." Acta Neurochirurgica. 2013; 155 (1): 71-74. Disponible en: https:// doi:10.1007/s00701-012-1553-4.

10- Torrens M, Chung C, Chung HT, Hanssens P, Jaffray D, Kemeny A, et al. Standardization of terminology in stereotactic radiosurgery: Report from the Standardization Committee of the International Leksell Gamma Knife Society: special topic. J Neurosurg. 2014 Dec;121 Suppl:2-15. doi: 10.3171/2014.7.GKS141199.

11- Soltys SG, Milano MT, Xue J, Tomé WA, Yorke E, Sheehan J, et al. Stereotactic Radiosurgery for Vestibular Schwannomas: Tumor Control Probability Analyses and Recommended Reporting Standards. Int. j. radiat. oncol. biol. phys. 2021; 110(1): 100-111. Disponible en: https://doi. org/10.1016/j.jijrobp.2020.11.019

12- Pollock BE. Management of vestibular schwannomas that enlarge after stereotactic radiosurgery: treatment recommendations based on a 15 year experience. Neurosurgery. 2006; 58(2): 241-248. https://doi.org/10.1227/01.NEU.0000194833.66593.8B.

13- Lustgarten $L$. The impact of stereotactic radiosurgery in the management of neurofibromatosis type 2-related vestibular schwannomas. Surg Neurol Int. 2013 Apr 17;4(Suppl 3):S151-5. doi: 10.4103/21527806.110663

14- Carlson ML, Jacob JT, Pollock BE, Neff BA, Tombers NM, Driscoll CL, et al. Long-term hearing outcomes following stereotactic radiosurgery for vestibular schwannoma: patterns of hearing loss and variables influencing audiometric decline. Journal of neurosurgery. 2013; 118(3): 579-587. Disponible en: https://doi.org/10.3171/2012.9.JNS12919.

15- Roos DE, Potter AE, Zacest AC. Hearing preservation after low dose linac radiosurgery for acoustic neuroma depends on initial hearing and time. Radiother Oncol. 2011 Dec;101(3):420-4. doi: 10.1016/j. radonc.2011.06.035

16- Yang I, Sughrue ME, Han SJ, Aranda D, Pitts LH, Cheung SW, Parsa AT. A comprehensive analysis of hearing preservation after radiosurgery for vestibular schwannoma. J Neurosurg. 2010 Apr;112(4):8519. doi: 10.3171/2009.8.JNS0985.

17- Yomo S, Carron R, Thomassin JM, Roche PH, Régis J. Longitudinal analysis of hearing before and after radiosurgery for vestibular schwannoma. J Neurosurg. 2012 Nov;117(5):877-85. doi: 10.3171/2012.7.JNS10672.

18- Jacob JT, Carlson ML, Schiefer TK, Pollock BE, Driscoll CL, Link MJ. Significance of cochlear dose in the radiosurgical treatment of vestibular schwannoma: controversies and unanswered questions. Neurosurgery. 2014 May;74(5):466-74; discussion 474. doi: 10.1227/NEU.0000000000000299.

19- Chang SD, Gibbs IC, Sakamoto GT, Lee E, Oyelese A, Adler JR Jr. Staged stereotactic irradiation for acoustic neuroma. Neurosurgery. 2005 Jun; 56(6):1254-61; discussion 1261-3. doi: 10.1227/01. neu.0000159650.79833.2b.

20- Linskey ME. Hearing preservation in vestibular schwannoma stereotactic radiosurgery: what really matters? J Neurosurg. 2008 Dec;109 Suppl:129-36. doi: 10.3171/JNS/2008/109/12/S20.

\section{Aporte de cada autor al trabajo}

Leandro Ricagni: concepción y diseño, recolección de datos, análisis e interpretación de los resultados, redacción del trabajo.

Milexis Rivero: concepción y diseño, recolección de datos, análisis e interpretación de los resultados, redacción del trabajo.

Pablo Pereda: concepción y diseño del trabajo, revisión crítica del manuscrito.

Aldo Quarneti: concepción y diseño del trabajo, revisión crítica del manuscrito. 